\title{
Nerve growth factor is closely related to glucose metabolism, insulin sensitivity and insulin secretion in the second trimester: a case-control study in Chinese
}

Mengyang Tang ${ }^{1,2+}$, Mingjuan Luo ${ }^{1,2,3 \dagger}$, Wenqian Lu ${ }^{1,2}$, Rong Zhang ${ }^{4}$, Wei Liang ${ }^{3}$, Jianfen Gu ${ }^{3}$, Xuemei Yu ${ }^{1,2}$, Xueli Zhang ${ }^{1,2}$ and Cheng $\mathrm{Hu}^{1,2,4^{*}}$ (D)

\begin{abstract}
Objective: Inflammation-related factors have been shown to play a significant role throughout pregnancy. In this study, we aimed to explore the relationships between selected inflammatory cytokines and gestational diabetes (GDM) in Chinese pregnant women.

Design and methods: This was a 1:1 matched case-control study that included 200 pairs of subjects in the second trimester and 130 pairs of subjects in the third trimester. Serum levels of nerve growth factor (NGF), Interleukin-6 (IL-6), leptin, Interleukin-8 (IL-8), monocyte chemoattractant protein-1 (MCP-1), tumor necrosis factor-alpha (TNF-a) and Interleukin-1 beta (IL-1 $\beta$ ) were measured by enzyme immunoassay. The associations of these inflammatory factors with metabolic parameters were analysed.

Results: In the second trimester, GDM patients had higher NGF levels and lower IL-8 levels than did normal controls $(P<0.001$ and $P=0.015$, respectively). However, in the third trimester, only lower leptin levels were observed in the GDM group $(P=0.031)$. Additionally, in the second trimester, NGF levels were not only positively associated with fasting, 1-h and 2-h glucose levels and the area under curve of glucose, but also positively related to insulin sensitivity and secretion, as suggested by fasting insulin, homeostasis model assessment of insulin resistance (HOMA-IR) and homeostasis model assessment index of $\beta$-cell secretion (HOMA- $\beta$ ) (all $P<0.05)$. Moreover, IL- 6 and leptin levels were positively correlated with HOMA-IR and HOMA- $\beta$, and TNF-a levels were positively related to HOMA-IR (all $P<0.05$ ). Except for the relationships between NGF and HOMA- $\beta$ and TNF- $\alpha$ and HOMA-IR, the other correlations still existed even after adjusting for confounding factors (all $P<0.05$ ).
\end{abstract}

Conclusion: In addition to the positive associations of IL-6 and leptin with insulin resistance and secretion, NGF was higher in the GDM patients and strongly linked to glucose metabolism, insulin resistance and pancreatic $\beta$ cell function in Chinese pregnant women in the second trimester.

Keywords: Gestational diabetes, Glucose metabolism, Inflammatory cytokines, Nerve growth factor

*Correspondence: alfredhc@sjtu.edu.cn

${ }^{\dagger}$ Mengyang Tang and Mingjuan Luo have contributed equally to this work

${ }^{1}$ The Third School of Clinical Medicine, Southern Medical University, Guangzhou, China

Full list of author information is available at the end of the article

\section{Background}

Gestational diabetes mellitus (GDM) is a pathological state in which pancreatic $\beta$ cells cannot release adequate insulin to meet the increased insulin demand [1]. Just as type 2 diabetes (T2DM), environmental factors, genetic 
factors, and epigenetic modifications all contribute to the accelerating GDM epidemic [2, 3]. Currently, chronic insulin resistance, an indirect cause of impaired pancreatic $\beta$ cell function, is thought to be the major pathological feature of GDM [4-6]. However, explorations of the exact mechanism of insulin resistance in GDM are still limited, with most studies concentrating on T2DM. As one of the leading causes of T2DM, insulin resistance is strongly associated with inflammation [7-10]. In obese patients with T2DM, which feature with macrophage accumulation and chronic inflammation, insulin resistance can be found in many tissues, including adipose tissue, the liver and skeletal muscle $[8,9]$. To date, scientists have revealed that one of the central pathogenic mechanisms of insulin resistance is the modulation of inflammation-related factors, which affects the gene expression regulation and metabolism of some lipid-related factors involved in the insulin signalling pathway [11].

Given the close relationship between inflammation and insulin resistance, many studies have focused on the relationships between inflammation-related factors and GDM. A prospective study indicated that increased serum C-reactive protein (CRP) levels could positively predict the morbidity of GDM [12]. Recently, a casecontrol study from Inner Mongolia also found that there were positive associations of high sensitivity CRP, Interleukin-6 (IL-6), Interleukin-8 (IL-8) and the IL-6/IL-10 ratio with GDM [13]. Other nontypical inflammatory factors such as growth differentiation factor 15 (GDF-15) was also proved to have a close relationship with GDM [14]. These data provide evidence that inflammatory cytokines are important factors in the pathological processes of GDM.

However, studies of the exact relationships between inflammatory factors and some important metabolic indexes in Chinese GDM patients are still limited. Therefore, in this study, through systematic analyses of selected inflammatory cytokines and GDM in both the second and third trimesters, we aimed to explore the differences in serum levels of nerve growth factor (NGF), IL-6, leptin, IL-8, monocyte chemoattractant protein-1 (MCP-1), Interleukin-1 beta (IL-1 $\beta$ ) and tumor necrosis factor-alpha (TNF- $\alpha$ ) between GDM patients and normal controls, and to analyse the exact correlations of these cytokines with glucose metabolism, insulin resistance and pancreatic $\beta$ cell function.

\section{Methods}

\section{Study participants and clinical measurements}

All participants and their clinical measurements were described in our previous article [14]. In brief, according to age ( \pm 3 years), pregestational BMI $\left( \pm 3 \mathrm{~kg} / \mathrm{m}^{2}\right)$, and gestational week ( \pm 3 weeks), we included 200 GDM patients and 200 matched normal controls in the second trimester as well as 130 GDM patients and 130 matched normal controls in the third trimester. Women with autoimmune disease, thyroid disease, heart trouble, liver or kidney disease, tumors, hematopathy, and other known diseases affecting glucolipid metabolism were excluded from this study. For the GDM groups in the second and third trimesters, there were no significant differences in age or pregestational BMI, and the same was true for the normal glucose tolerance (NGT) groups (both $P>0.05$ ). This study was conformed to the provision of the Declaration of Helsinki and approved by The Medical Ethics Committee of University of Hong Kong Shenzhen Hospital. We have obtained written informed consent from every participant before undertaking this study.

\section{Measurement of serum inflammatory cytokines}

NGF, IL-6, leptin, IL-8, MCP-1, TNF- $\alpha$ and IL- $1 \beta$ were detected using a MILLIPLEX ${ }^{\circledR}$ MAP kit (Merck Corp., New Jersey, USA) on a MAGPIX analyser (Merck Corp.). This assay has been proven to be highly sensitive for all inflammatory cytokines, with minimum detectable doses of human NGF, IL- 6 , leptin, IL-8, MCP-1, TNF- $\alpha$ and IL- $1 \beta$ of $0.3,0.2,3.8,19,1.2,0.3$ and $0.4 \mathrm{pg} / \mathrm{ml}$, respectively. No or negligible cross-reactivity of these antibodies across the seven inflammatory cytokines was detected. Moreover, except for the inter-assay variation of TNF- $\alpha$, which was less than $20 \%$, the intra-assay variations and inter-assay variations for all of these cytokines were less than $10 \%$ and less than $15 \%$, respectively.

\section{Statistical analysis}

SAS (version 8.0) was used to perform statistical analyses. GraphPad Prism software (version 7.0) was used to plot the graphs. All of the data were subjected to a normality test. If the data were normally distributed, they were expressed as the mean \pm SD. Data with an abnormal distribution were expressed as the median with the interquartile range and $\log 10$ transformed when analysed. According to the normality of the variables involved in the analysis, comparisons between two groups were conducted by a Student's paired $t$ test or sign-rank test, and relationships of inflammatory cytokines with metabolism parameters were estimated by Spearman or Pearson correlation coefficients. Categorical variables were analysed by the chi-square test. Spearman partial correlation analysis was used to adjust for confounding factors, including age, pregestational BMI, changes of BMI, gestational age, blood pressure, previous history of GDM, family history of diabetes and all the inflammatory cytokines. Results were considered statistically significant if two-tailed $P<0.05$. 


\section{Results}

\section{Characteristics of subjects}

The clinical characteristics of all participants have been described previously [14]. In brief, in addition to the increased blood pressure, fasting insulin, homeostasis model assessment of insulin resistance (HOMA-IR) and $\beta$-cell secretion (HOMA- $\beta$ ) in the GDM groups in the second trimester, GDM patients in both the second and third trimesters had higher previous history of GDM, family history of diabetes, HbA1c, fasting, 1-h and 2-h glucose levels and greater area under curve of glucose (AUCG) values than did the normal controls (all $P<0.05$ ) (Table 1).

\section{Inflammatory cytokine levels of the GDM and NGT groups in the second and third trimesters}

We compared the levels of the seven inflammatory factors between the GDM and NGT groups in both the second and third trimesters. The results showed that in the second trimester, serum NGF levels of GDM patients were remarkably higher than those of the normal controls $(P<0.001)$ (Table 2 and Fig. 1$)$, whereas GDM patients had lower IL-8 levels $(P=0.015)$. However, in the third trimester, NGF and IL-8 no longer showed significant differences, while lower leptin levels were observed in the GDM group than in the NGT group (Table 2 and Fig. 1).

\section{Associations of selected inflammatory cytokines with glucose metabolism, insulin resistance and pancreatic $\beta$ cell function}

Because of the missing data related to glucose levels in the third trimester, we conducted correlation analysis for only the second trimester data. When we divided our samples into 2 groups according to inflammatory levels, we found higher NGF levels were associated with a higher risk of GDM even after adjusting for confounding factors $(\mathrm{OR}(95 \% \mathrm{CI})=6.71(4.02,11.18)$, $P<0.0001)$. Besides, the glucose levels, insulin levels, insulin resistance and secretion also increased with the increasing NGF levels (Additional file 1: Table S1). Next, we conducted correlation analysis, and the results showed that NGF was not only positively associated with fasting $(P=0.007), 1-\mathrm{h}(P<0.001)$ and 2 -h glucose levels $(P<0.001)$ and AUCG values $(P<0.001)$, but also positively related to insulin resistance and pancreatic $\beta$ cell function, as shown by fasting insulin $(P<0.001)$ HOMA-IR $\quad(P<0.001)$ and HOMA- $\beta \quad(P=0.043)$ (Fig. 2). The other inflammation-related factors, such

Table 1 Characteristics of the GDM and NGT groups in both the second and third trimesters

\begin{tabular}{|c|c|c|c|c|c|c|}
\hline \multirow[t]{2}{*}{ Characteristics } & \multicolumn{3}{|c|}{ Participants in the second trimester } & \multicolumn{3}{|c|}{ Participants in the third trimester } \\
\hline & $\begin{array}{l}\text { GDM } \\
(n=200)\end{array}$ & $\begin{array}{l}\text { NGT } \\
(n=200)\end{array}$ & $P$ & $\begin{array}{l}\text { GDM } \\
(n=130)\end{array}$ & $\begin{array}{l}\text { NGT } \\
(n=130)\end{array}$ & $P$ \\
\hline Age (years) & $32(29,35)$ & $32(29,35)$ & 0.906 & $31(29,34)$ & $31(29,33)$ & 0.897 \\
\hline $\mathrm{SBP}$ (mmhg) & $109.83 \pm 10.14$ & $106(101,113)$ & 0.005 & & & \\
\hline $\mathrm{DBP}(\mathrm{mmhg})$ & $67.44 \pm .80$ & $65(61,71)$ & 0.022 & & & \\
\hline Pregestational BMI (kg/m²) & $21.32 \pm 2.59$ & $21.26 \pm 2.52$ & 0.343 & $21.19 \pm 2.29$ & $21.06 \pm 2.15$ & 0.695 \\
\hline Normal weight, n (\%) & $171(85.5)$ & $173(86.5)$ & & $112(86.15)$ & $118(90.77)$ & \\
\hline Overweight, n (\%) & $24(12)$ & $23(11.5)$ & 0.93 & $18(13.85)$ & $12(9.23)$ & 0.24 \\
\hline Obesity, n (\%) & $5(2.5)$ & $4(2)$ & & 0 & 0 & \\
\hline Changes of BMI $\left(\mathrm{kg} / \mathrm{m}^{2}\right)$ & $2.69 \pm 1.23$ & $2.70 \pm 1.23$ & 0.792 & $5.15 \pm 1.66$ & $5.78 \pm 1.35$ & 0.001 \\
\hline Previous history of GDM, n (\%) & $19(9.5)$ & $1(0.5)$ & $<0.001$ & $12(9.23)$ & $3(2.31)$ & 0.017 \\
\hline Family history of diabetes, n (\%) & $48(24)$ & $5(2.5)$ & $<0.001$ & $37(18.5)$ & $23(11.5)$ & 0.039 \\
\hline Gestational age (weeks) & $26(25,27)$ & $25(25,26)$ & 0.498 & $37(36,37)$ & $36(36,37)$ & 0.024 \\
\hline $\mathrm{HbA1c}(\%)$ & $5.20(5.00,5.40)$ & $5.10(5.00,5.30)$ & 0.003 & $5.40(5.20,5.60)$ & $5.20(5.00,5.40)$ & $<0.001$ \\
\hline $\mathrm{FBG}(\mathrm{mmol} / \mathrm{L})$ & $4.64 \pm 0.53$ & $4.36 \pm 0.28$ & $<0.001$ & $4.70 \pm 0.54$ & $4.37 \pm 0.28$ & $<0.001$ \\
\hline 1h-PG (mmol/L) & $9.88 \pm 1.32$ & $7.45 \pm 1.24$ & $<0.001$ & $9.71 \pm 1.61$ & $7.16 \pm 1.43$ & $<0.001$ \\
\hline 2h-PG (mmol/L) & $9.07 \pm 1.24$ & $6.66 \pm 0.98$ & $<0.001$ & $8.55 \pm 1.42$ & $6.34 \pm 1.01$ & $<0.001$ \\
\hline AUCG (mmol/L h) & $16.74 \pm 1.72$ & $12.97 \pm 1.56$ & $<0.001$ & $16.33 \pm 2.07$ & $12.52 \pm 1.77$ & $<0.001$ \\
\hline Fasting insulin (mU/L) & $6.79(4.75,10.78)$ & $5.37(3.73,7.77)$ & 0.001 & & & \\
\hline HOMA-IR & $1.38(0.95,2.21)$ & $1.05(0.71,1.53)$ & $<0.001$ & & & \\
\hline HOMA- $\beta$ & $134.07(88.68,206.35)$ & $123.20(81.80,215.58)$ & 0.522 & & & \\
\hline
\end{tabular}

Data are presented as the means $\pm S D$ s or median (interquartile range)

$S B P$ systolic blood pressure, $D B P$ diastolic blood pressure, $B M /$ body mass index, $F B G$ fasting blood glucose, $1 h$-PG 1-h postprandial glucose, $2 h$ - $P G$ 2-h postprandial glucose, AUCG area under curve of glucose from the 75-g OGTT, HOMA-IR homeostasis model assessment of insulin resistance, $H O M A-\beta$ homeostasis model assessment index of $\beta$-cell secretion 
Table 2 Inflammatory cytokines in the second and third trimesters

\begin{tabular}{|c|c|c|c|c|c|c|}
\hline & \multicolumn{3}{|c|}{ Subjects in the second trimester } & \multicolumn{3}{|c|}{ subjects in the third trimester } \\
\hline & GDM $(n=200)$ & NGT $(n=200)$ & $P$ & GDM $(n=130)$ & NGT $(n=130)$ & $P$ \\
\hline NGF (pg/ml) & $2.92(2.05,3.78)$ & $1.87(1.42,2.43)$ & $<0.001$ & $1.86(1.33,2.41)$ & $1.97(1.51,2.64)$ & 0.055 \\
\hline IL-6 (pg/ml) & $3.69(2.01,7.82)$ & $4.28(2.36,7.84)$ & 0.217 & $4.32(2.53,9.21)$ & $4.33(2.53,9.4)$ & 0.838 \\
\hline Leptin (ng/ml) & $10.74(7.28,13.79)$ & $11.23(7.08,16.00)$ & 0.099 & $10.33(6.46,13.53)$ & $11.91(7.94,17.39)$ & 0.031 \\
\hline IL-8 (pg/ml) & $101.23(42.03,202.40)$ & $126.46(47.84,268.77)$ & 0.015 & $33.56(17.58,77.18)$ & $34.89(17.12,57.27)$ & 0.159 \\
\hline MCP-1 (pg/ml) & $146.8(109.86,189.25)$ & $158.37(112.18,211.55)$ & 0.059 & $142.48(114.32,173.62)$ & $145.73(112.77,185.09)$ & 0.866 \\
\hline TNF-a (pg/ml) & $3.33(2.39,4.44)$ & $3.22(2.23,4.28)$ & 0.432 & $3.94(2.46,5.59)$ & $3.53(2.28,4.79)$ & 0.121 \\
\hline $\mathrm{IL}-1 \beta(\mathrm{pg} / \mathrm{ml})$ & $1.78(1.13,3.95)$ & $2.15(1.13,4.45)$ & 0.271 & $1.09(1.01,1.97)$ & $1.09(1.01,1.49)$ & 0.260 \\
\hline
\end{tabular}

Data are median (interquartile range)

GDM gestational diabetes mellitus, NGT normal glucose tolerance, NGF nerve growth factor, IL-6 Interleukin-6, IL-8 Interleukin-8, MCP- monocyte chemoattractant protein 1,TNFa tumor necrosis factor-alpha, IL- $\beta$ Interleukin-1 beta
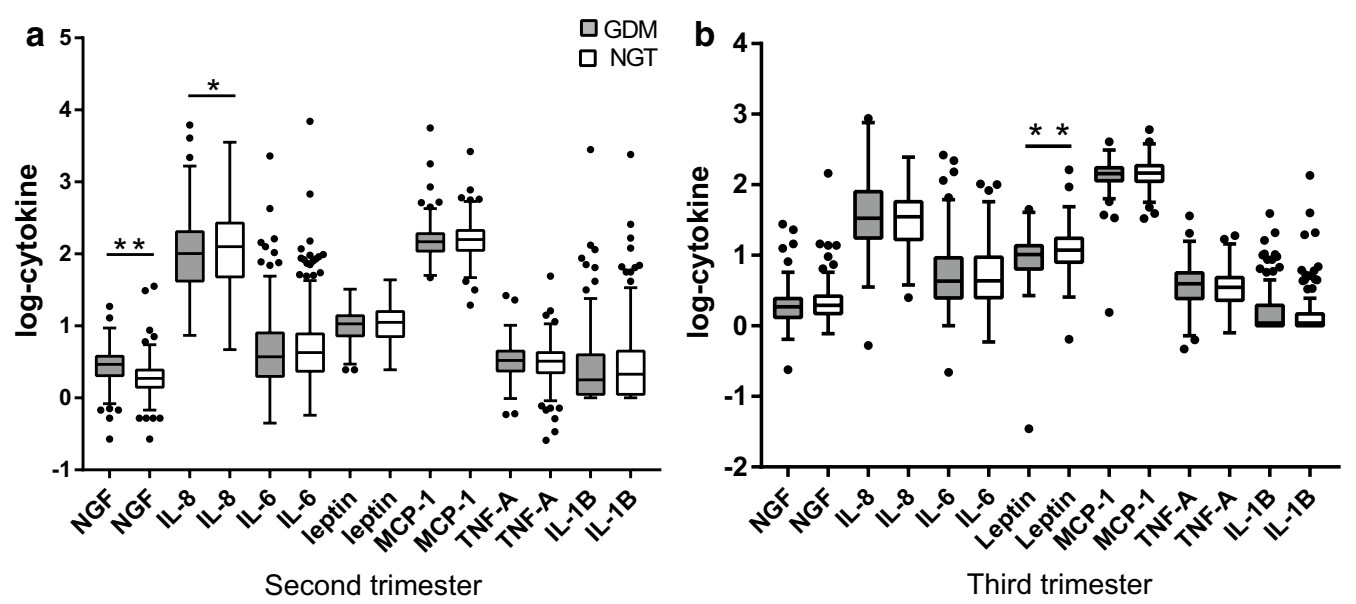

Fig. 1 Serum levels of selected inflammatory cytokines in the second and third trimesters. The horizontal lines represent the medians, the tops and bottoms of the boxes represent quartiles, and the t-bars denote the 25th and 75th percentiles plus and minus the 1.5 interquartile distances. The solid circles represent outliers. ${ }^{*} P<0.05 ;{ }^{* *} P<0.001$. NGF nerve growth factor, IL-8 Interleukin-8, IL-6 Interleukin-6, MCP-1 monocyte chemoattractant protein 1, TNF- $a$ tumor necrosis factor-alpha, IL- $\beta$ Interleukin-1 beta

as IL-6 and leptin, were positively correlated with only HOMA-IR and HOMA- $\beta$ (all $P<0.001$ ), and TNF- $\alpha$ was positively related to only HOMA-IR $(P=0.022)$ (Fig. 3). Except for the relationships between TNF- $\alpha$ and HOMA-IR and NGF and HOMA- $\beta$, the other relationships still existed even after adjusting for maternal age, pregestational BMI, gestational age, changes of BMI, blood pressure, family history of diabetes, previous history of GDM and all the other inflammatory cytokines (all $P<0.05$ ). As women who were overweight and obese have a higher chance of being diabetic or having other metabolic dysfunction, we also conducted correlation analysis after excluding them from our study and reanalyzed the data. As expected, the new result showed the same trends just as we got above (Additional file 2: Table S2).

\section{Discussion}

There have been many studies assessing the exact changes in inflammatory cytokines throughout normal gestation, but explorations of the relationships among these cytokines and metabolic indexes in Chinese pregnant women are still limited. Through our investigation, in addition to the positive associations of leptin, IL-8 and TNF- $\alpha$ with insulin resistance or secretion, NGF was found for the first time to be higher in GDM patients and positively associated with fasting and postprandial glucose metabolism, insulin resistance and pancreatic $\beta$ cell function in the second trimester in Chinese.

NGF is an essential factor for the development of the nervous system in individuals and is necessary for healthy pregnancies $[15,16]$. In addition, NGF is an inflammatory factor that can be induced by a variety 

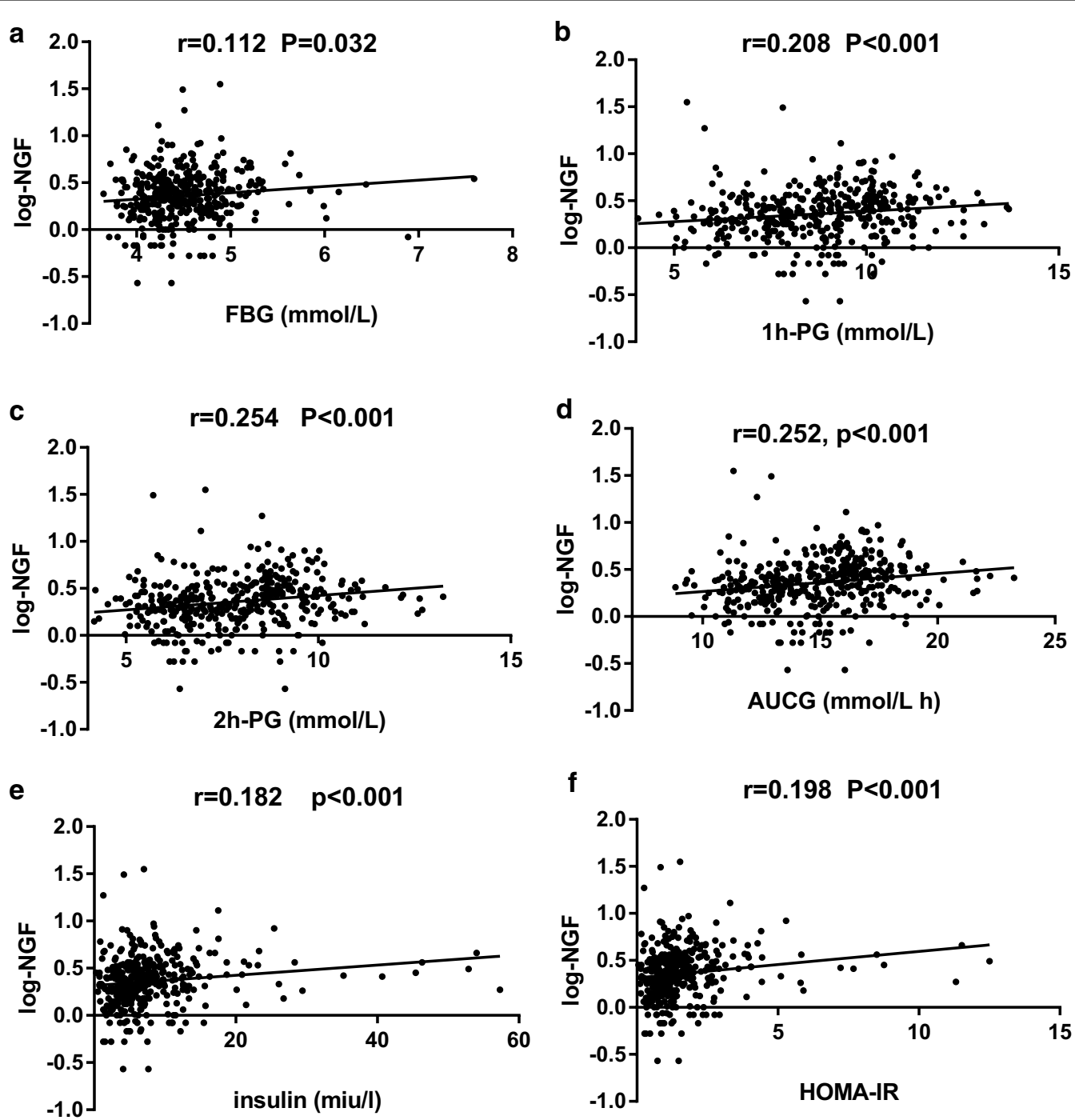

Fig. 2 Correlations of NGF with glucose levels, insulin resistance and insulin secretion. $r$ and p: adjusted for all the cytokines, maternal age, gestational age, pregestational BMI, changes of BMl, blood pressure, family history of diabetes and previous history of GDM. NGF nerve growth factor, FBG fasting blood glucose, 1 h-PG 1-h postprandial glucose, 2h-PG 2-h postprandial glucose, AUCG area under curve of glucose from the 2-h OGTT, HOMA-IR homeostasis model assessment of insulin resistance, HOMA- $\beta$ homeostasis model assessment index of $\beta$-cell secretion

of inflammatory diseases [17]. In many articles, NGF expression was shown to be increased in diabetes mellitus and it has been proven to be an important protective factor in diabetic neuropathy and vasculopathy [18-21]. We suspected that the vascular and neuroprotective effects of NGF also played significant roles in the physiological processes of GDM. In our study, serum levels of NGF were significantly higher in GDM patients than in normal controls, suggesting the essential role of NGF in GDM. However, the levels of NGF in this study were different from previous reports [22-24]. The difference of sample types might be part of the reasons as most of those studies were conducted with the plasm while we used the serum. In addition, the method we used which could detect all of seven inflammatory factors in one panel is also different from the traditional ELISA method.

Currently, studies have found that NGF and its receptor Tyrosine kinase $A$ ( $\operatorname{Tr} k A)$ are expressed in many tissues, including $\beta$ cells of the pancreas [25-27], and that high glucose levels could dramatically enhance the secretion of NGF and subsequently activate $\beta$-cell TrkA receptors to acutely promote glucose-stimulated insulin secretion $[28,29]$. These results were consistent with our finding that NGF was positively related to glucose levels and insulin secretion in the second trimester. Moreover, NGF could reduce insulin resistance both in vitro and 

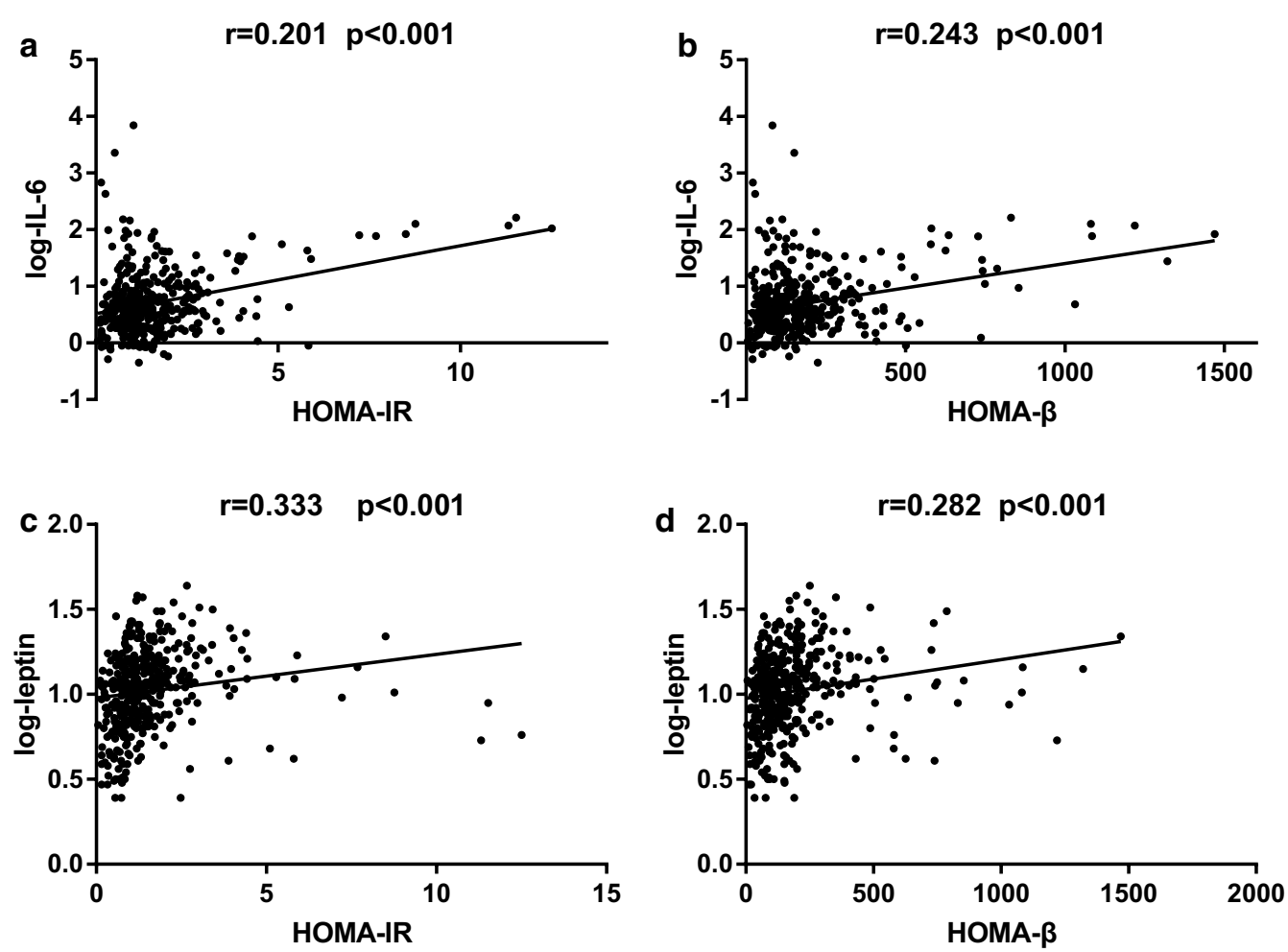

Fig. 3 Correlations of IL-6 and leptin with insulin secretion and resistance. $r$ and p: adjusted for all the cytokines, maternal age, gestational age, pregestational BMI, changes of BMI, blood pressure, family history of diabetes and previous history of GDM. IL-6 Interleukin-6, HOMA-IR homeostasis model assessment of insulin resistance, HOMA- $\beta$ homeostasis model assessment index of $\beta$-cell secretion

in vivo by activating insulin receptor substrate 1 (IRS1) [30, 31]. Therefore, NGF levels are supposed to have a negative relationship with insulin resistance. However, in our study, we found NGF levels were positively associated with insulin resistance, which might be ascribed to the compensatory adjustment. These data suggest that NGF acts as a protective factor in glucose metabolism and insulin sensitivity and plays a significant role in glucose-induced insulin secretion. Although the correlations between NGF and these metabolism indexes were poor as all $\mathrm{r}<0.3$, given the significant role of NGF in insulin signal pathway, and the close relationship between NGF and GDM, we could not neglect the essential role of NGF in GDM.

Compared to the increasing NGF levels and decreasing IL-8 levels in GDM patients in the second trimester, in the third trimester, only leptin levels showed a significant difference between the GDM group and NGT group. Considering the close relationships between glucose levels and inflammatory responses [32], we speculated that the different results might be due to the diverse glucose metabolism states of the participants, as $16.7 \%$ of the GDM patients in the third trimester had been treated with insulin. Unfortunately, there is no possibility to study the rest patients who didn't receive insulin therapy on account of the lack of data on glucose and insulin levels in the third trimester. Additionally, participants in the second and third trimesters were different, although they shared the same origin and showed no significant differences in age and pregestational BMI, we could not exclude the possibility of population heterogeneity.

Correlations of IL-6, leptin and TNF- $\alpha$ with diabetes have been explored extensively, and most of these studies obtained results similar to ours that TNF- $\alpha$, IL- 6 and leptin were positively associated with insulin resistance or secretion [33, 34]. However, after adjustments, the relationship between TNF- $\alpha$ and insulin resistance disappeared. In addition, no relationships were found between MCP-1 or IL- 8 and glucose metabolism or insulin resistance and secretion, which was contradictory to the consequences of some studies [33, 35]. In this study, serum levels of IL-8 were statistical significance between GDM patients and normal controls but showed no relationships with glucose metabolism indexes or insulin secretion/resistance. However, we could not rule out the possibility that there were associations between IL-8 and other metabolism indexes causing the differences, although we did not have other 
clinical metabolism indexes to perform the correlation analysis. In addition, the special physiological status of GDM and the combined action of diverse diets, lifestyles and gene-profiles might partly account for these differences.

Our limitations are as follows: First, the study was a case-control study that could not explore the changes in serum inflammatory cytokine levels throughout the course of pregnancy. Moreover, in the third trimester, due to missing data on glucose levels and insulin secretion, no investigation of the relationships of inflammatory cytokines and glucose metabolism was conducted. Last, our participants in the second and third trimesters were from two different populations, and we could not determine if the differences in the second and third trimesters resulted from GDM therapy or population heterogeneity. Further longitudinal cohort and large sample size studies with more comprehensive data are still needed to explore which inflammation-related factors are involved in the progression of GDM throughout pregnancy.

\section{Conclusions}

In conclusion, this systematic study investigated the relationships of selected inflammatory cytokines and GDM. We found that serum levels of NGF was higher in GDM patients in the second trimester, but not in the third trimester. Moreover, in the second trimester, in addition to the positive associations of IL-6 and leptin with insulin resistance, NGF was closely related to glucose metabolism, insulin resistance and pancreatic $\beta$ cell function and might be a protective factor in the pathological process of GDM.

\section{Supplementary information}

Supplementary information accompanies this paper at https://doi. org/10.1186/s12986-020-00523-2.

Additional file 1: Table S1. The relationships between inflammatory factors and clinical indexes.

Additional file 2: Table S2. Correlations of inflammatory factors and metabolism indexes after excluded women who were overweight or obese.

\begin{abstract}
Abbreviations
AUCG: Area under curve of glucose from the $75-g$ oral glucose tolerance test; BMI: Body mass index; DBP: Diastolic blood pressure; FBG: Fasting blood glucose; GDM: Gestational diabetes mellitus; HOMA-IR: Homeostasis model assessment of insulin resistance; HOMA- $\beta$ : Homeostasis model assessment index of $\beta$-cell secretion; IL-6: Interleukin-6; IL-8: Interleukin-8; IL- $\beta$ : Interleukin-1 beta; MCP-1: Monocyte chemoattractant protein 1; NGT: Normal glucose tolerance; NGF: Nerve growth factor; OGTT: Oral glucose tolerance test; SBP: Systolic blood pressure; TNF-a: Tumor necrosis factor-alpha; 1h-PG: 1-hour postprandial glucose; 2h-PG: 2-hour postprandial glucose.
\end{abstract}

Acknowledgements

Not applicable.

\section{Authors' contributions}

$\mathrm{CH}$ designed the study. MT performed the experiments, statistical analysis and wrote the manuscript. MT and ML collected and checked the data; WL and RZ helped to perform the experiment. $X Y$ and $X Z$ reviewed the manuscript. $\mathrm{CH}$ reviewed and revised the manuscript. All authors have read and approved the final manuscript.

\section{Funding}

This work was supported by the National Key Research and Development Project of China (2016YFC1304902), National Science Foundation of China (81570713 and 91649112), Outstanding Academic Leaders of Shanghai Health System (2017BR008), the National Program for Support of Top-notch Young Professionals and Yangtze River Scholar, The University of Hong Kong-Shenzhen Hospital "High-level Hospital" Construction Scientific Research Cultivation Plan (201901025).

\section{Availability of data and materials}

The datasets analyzed during our current study are not publicly available but are available from the corresponding author on reasonable request.

\section{Ethics approval and consent to participate}

This study was conformed to the provision of the Declaration of Helsinki and approved by The Medical Ethics Committee of University of Hong Kong Shenzhen Hospital. We have obtained written informed consent from every participant before undertaking this study.

\section{Consent for publication}

Not applicable.

\section{Competing interests}

The authors declare no conflict of interest.

\section{Author details \\ ${ }^{1}$ The Third School of Clinical Medicine, Southern Medical University, Guang- zhou, China. ${ }^{2}$ Department of Endocrinology and Metabolism, Fengxian Central Hospital Affiliated to the Southern Medical University, Shanghai, China. ${ }^{3}$ Department of Endocrinology and Metabolism, University of Hong Kong Shenzhen Hospital, Shenzhen, China. ${ }^{4}$ Shanghai Diabetes Institute, Shanghai Jiao Tong University Affiliated Sixth People's Hospital, Shanghai, China.}

Received: 10 July 2020 Accepted: 10 November 2020

Published online: 19 November 2020

\section{References}

1. Reece EA, Leguizamon G, Wiznitzer A. Gestational diabetes: the need for a common ground. Lancet. 2009;373(9677):1789-97.

2. Mclntyre HD, et al. Gestational diabetes mellitus. Nat Rev Dis Primers. 2019;5(1):47.

3. Hu C, Jia W. Diabetes in China: epidemiology and genetic risk factors and their clinical utility in personalized medication. Diabetes. 2018;67(1):3-11.

4. Buchanan TA, Xiang AH. Gestational diabetes mellitus. J Clin Investig. 2005;115(3):485-91.

5. Plows JF, et al. The pathophysiology of gestational diabetes mellitus. Int J Mol Sci. 2018;19(11):3342.

6. Du X, et al. Association between retinol-binding protein 4 concentrations and gestational diabetes mellitus (A1GDM and A2GDM) in different pregnancy and postpartum periods. Ann Transl Med. 2019;7(18):479.

7. Siwicki M, Engblom C, Pittet MJ. Gal3 links inflammation and insulin resistance. Cell Metab. 2016;24(5):655-6.

8. Kahn SE, Hull RL, Utzschneider KM. Mechanisms linking obesity to insulin resistance and type 2 diabetes. Nature. 2006;444(7121):840-6.

9. Cao W, et al. Adipocytes initiate an adipose-cerebral-peripheral sympathetic reflex to induce insulin resistance during high-fat feeding. Clin Sci (Lond Engl). 2019;133(17):1883-99.

10. Lou M, et al. Relationship between neutrophil-lymphocyte ratio and insulin resistance in newly diagnosed type 2 diabetes mellitus patients. BMC Endocr Disord. 2015;15:9. 
11. Lackey DE, Olefsky JM. Regulation of metabolism by the innate immune system. Nat Rev Endocrinol. 2016;12(1):15-28.

12. Wolf $M$, et al. First-trimester $C$-reactive protein and subsequent gestational diabetes. Diabetes Care. 2003;26(3):819-24.

13. Zhang J, et al. Interleukin 6 (IL-6) and tumor necrosis factor alpha (TNF-alpha) single nucleotide polymorphisms (SNPS), inflammation and metabolism in gestational diabetes mellitus in inner mongolia. Med Sci Monit. 2017;23:4149-57.

14. Tang $M$, et al. Serum growth differentiation factor 15 is associated with glucose metabolism in the third trimester in Chinese pregnant women. Diabetes Res Clin Pract. 2019;156:107823.

15. Lindsay RM, Harmar AJ. Nerve growth factor regulates expression of neuropeptide genes in adult sensory neurons. Nature. 1989;337(6205):362-4.

16. Frank $P$, et al. Balanced levels of nerve growth factor are required for normal pregnancy progression. Reproduction. 2014;148(2):179-89.

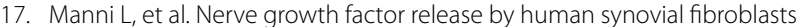
prior to and following exposure to tumor necrosis factor-alpha, interleukin-1 beta and cholecystokinin-8: the possible role of NGF in the inflammatory response. Clin Exp Rheumatol. 2003;21(5):617-24.

18. Azar ST, Major SC, Safieh-Garabedian B. Altered plasma levels of nerve growth factor and transforming growth factor-beta2 in type-1 diabetes mellitus. Brain Behav Immun. 1999;13(4):361-6.

19. Ali TK, et al. Peroxynitrite mediates retinal neurodegeneration by inhibiting nerve growth factor survival signaling in experimental and human diabetes. Diabetes. 2008;57(4):889-98.

20. Li R, et al. Heparin-poloxamer thermosensitive hydrogel loaded with bFGF and NGF enhances peripheral nerve regeneration in diabetic rats. Biomaterials. 2018;168:24-37.

21. Li R, et al. Dual delivery of NGF and bFGF coacervater ameliorates diabetic peripheral neuropathy via inhibiting schwann cells apoptosis. Int J Biol Sci. 2017:13(5):640-51.

22. D'Souza $V$, et al. Maternal nerve growth factor levels during pregnancy in women with preeclampsia: a longitudinal study. Int J Dev Neurosci Off J Int Soc Dev Neurosci. 2015;47(Pt B):340-6.

23. Sepúlveda-Martínez A, et al. Maternal plasma nerve growth factor at the $11+0-13+6$ weeks' scan as a potential angiogenic marker of preeclampsia: a pilot study. Fetal Diagn Ther. 2017;41(3):202-8
24. Dhobale $\mathrm{M}$, et al. Reduced maternal and cord nerve growth factor levels in preterm deliveries. Int J Dev Neurosci Off J Int Soc Dev Neurosci. 2012;30(2):99-103.

25. Tessarollo L. Pleiotropic functions of neurotrophins in development. Cytokine Growth Factor Rev. 1998;9(2):125-37.

26. Kanaka-Gantenbein C, et al. Presence of nerve growth factor and its receptors in an in vitro model of islet cell development: implication in normal islet morphogenesis. Endocrinology. 1995;136(7):3154-62.

27. Rosenbaum T, et al. Pancreatic beta cells synthesize and secrete nerve growth factor. Proc Natl Acad Sci U S A. 1998;95(13):7784-8.

28. Houtz J, et al. Neurotrophin signaling is required for glucose-induced insulin secretion. Dev Cell. 2016;39(3):329-45.

29. Pingitore $A$, et al. Fine tuning of insulin secretion by release of nerve growth factor from mouse and human islet beta-cells. Mol Cell Endocrinol. 2016;436:23-32.

30. Gezginci-Oktayoglu S, Karatug A, Bolkent $\mathrm{S}$. The relation among NGF, EGF and insulin is important for triggering pancreatic beta cell apoptosis. Diabetes Metab Res Rev. 2012;28(8):654-62.

31. Sposato $V$, et al. The medial septum is insulin resistant in the AD presymptomatic phase: rescue by nerve growth factor-driven IRS1 activation. Mol Neurobiol. 2019;56(1):535-52.

32. Chang SC, Yang WV. Hyperglycemia, tumorigenesis, and chronic inflammation. Crit Rev Oncol Hematol. 2016;108:146-53.

33. Degirmenci I, Ozbayer C. Common variants of genes encoding TLR4 and TLR4 pathway members TIRAP and IRAK1 are effective on MCP1, IL6, IL1 beta, and TNFalpha levels in type 2 diabetes and insulin resistance. Inflamm Res. 2019:68(9):801-14.

34. Akash MSH, Rehman K, Liaqat A. Tumor necrosis factor-alpha: role in development of insulin resistance and pathogenesis of type 2 diabetes mellitus. J Cell Biochem. 2018;119(1):105-10.

35. Zheng $X$, et al. The mechanism by which amentoflavone improves insulin resistance in HepG2 cells. Molecules. 2016;21(5):624.

\section{Publisher's Note}

Springer Nature remains neutral with regard to jurisdictional claims in published maps and institutional affiliations.
Ready to submit your research? Choose BMC and benefit from:

- fast, convenient online submission

- thorough peer review by experienced researchers in your field

- rapid publication on acceptance

- support for research data, including large and complex data types

- gold Open Access which fosters wider collaboration and increased citations

- maximum visibility for your research: over 100M website views per year

At BMC, research is always in progress.

Learn more biomedcentral.com/submissions 